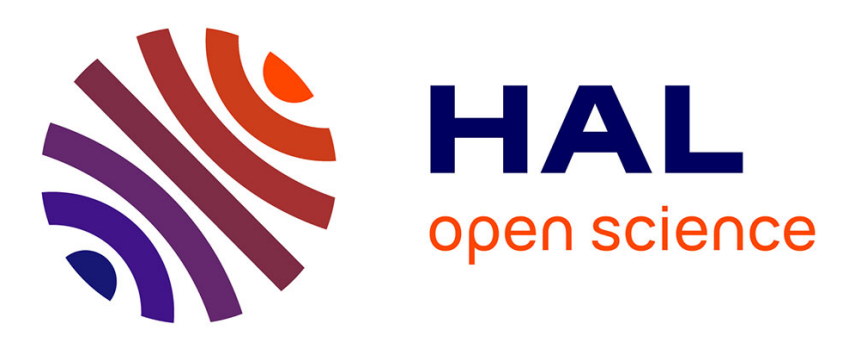

\title{
Surface defects sensitivity during the unfolding of corrugated struts made by powder-bed Additive Manufacturing
}

Mathieu Suard, Emeric Plancher, Guilhem Martin, Rémy Dendievel, Pierre Lhuissier

\section{To cite this version:}

Mathieu Suard, Emeric Plancher, Guilhem Martin, Rémy Dendievel, Pierre Lhuissier. Surface defects sensitivity during the unfolding of corrugated struts made by powder-bed Additive Manufacturing. Advanced Engineering Materials, 2020, 10.1002/adem.202000315 . hal-03021833

\section{HAL Id: hal-03021833 https://hal.science/hal-03021833}

Submitted on 24 Nov 2020

HAL is a multi-disciplinary open access archive for the deposit and dissemination of scientific research documents, whether they are published or not. The documents may come from teaching and research institutions in France or abroad, or from public or private research centers.
L'archive ouverte pluridisciplinaire HAL, est destinée au dépôt et à la diffusion de documents scientifiques de niveau recherche, publiés ou non, émanant des établissements d'enseignement et de recherche français ou étrangers, des laboratoires publics ou privés. 


\title{
Surface defects sensitivity during the unfolding of corrugated struts made by powder-bed Additive Manufacturing.
}

\author{
Mathieu Suard*, Emeric Plancher, Guilhem Martin, Rémy Dendievel, Pierre Lhuissier
}

Dr. M. Suard, Dr. E. Plancher, Dr. G. Martin, Pr. R. Dendievel, Dr. P. Lhuissier

Univ. Grenoble Alpes, CNRS, Grenoble INP, SIMaP,

F-38000 Grenoble (France)

E-mail: mathieu.suard.am@gmail.com,

Keywords: Powder-bed Additive Manufacturing; surface defects; in-situ micro-CT tensile test; micromechanics; probabilistic model.

\begin{abstract}
Corrugated struts as part of lattice structures can lead to novel mechanical behavior by a combination of material and geometrical hardening. The unfolding behavior of such struts offers a potential of large macroscopic straining. However, their ability to be unfolded is impacted by the surface characteristics inherited from the additive manufacturing process. This study evaluates the unfolding sensitivity to these surface characteristics. Corrugated struts with varying surface roughness have been produced using a combination of Electron Beam Powder-Bed Fusion to produce corrugated samples with different nominal diameters, and chemical etching assisted by micro-CT to achieve a given final diameter. In-situ micro-CT tensile tests have been carried out to track the evolution of the struts morphology under loading. Surface defects play a significant role in the unfolding ability of such struts. They are characterized either by a global roughness or by a local notch depth. A quite broad unfolding dispersion remains for samples with the same level of roughness. A finer description of notch depth and location within the gauge length allows a more accurate prediction of the unfolding ability. A model for predicting the probability of failure during unfolding is presented.
\end{abstract}




\section{Introduction}

Developing materials by design to fulfill multi-criteria sets of requirements has been proven for some years now, see e.g. ${ }^{[1]-[4]}$. In order to expand the range of properties of monolithic materials, several design variables can be tuned as explained by Ashby in ${ }^{[4]}$. Designer can play with constitutive materials and architecture (geometry, topology). This strategy has paved the way to lattice ${ }^{[2] 5]}$ and composite structures ${ }^{[6]}$, macro-micro structure optimization ${ }^{[7][8]}$. In a recent example of such a strategy Pham et al. ${ }^{[9]}$ manufactured architectured materials with a mesostructure inspired by crystal microstructure that exhibit unconventional mechanical properties. Using analogies with crystal orientations, precipitates, grain boundaries at the mesoscale of the lattice structure, they created robust and damage-tolerant structures.

Here, we focus on adding new mechanical features to a material by playing with its shape. The aim is to produce a lattice structure with optimized geometry for each strut. The use of corrugated struts has been proven to improve the work-hardening rate of the structure by creating geometrically-induced strain hardening ${ }^{[10][11]}$. Plancher et al. ${ }^{[12]}$ recently developed a toolbox to predict the requested characteristics of corrugated samples based on the expected stress-strain response. The use of an elastic-plastic simulation allowed the period, amplitude and thickness of corrugated planes to be optimized. Such samples were built using the Electron Beam Powder-Bed Fusion (E-PBF) additive manufacturing technique.

Additive manufacturing is particularly well suited to produce complex shapes. It allows a larger freedom in designing parts but comes along with some limitations. Inherited defects such as a large surface roughness, a variable dimensional accuracy and a characteristic porosity have been widely studied, see e.g. ${ }^{[13]-[16]}$. The as-built surface can be detrimental for lattice structures for which no machining can be used. The discrimination of most detrimental defects for parts fabricated by E-PBF under tensile loading has been studied for fatigue applications, see ${ }^{[17]}$. The use of micro-CT prior and post-loading explained the reduced fatigue properties by taking into account the depth and width of the notch-like defects.

Reducing the surface defect harmfulness is of primary importance when producing structural parts. To improve the mechanical properties of titanium lattice structures additively manufactured, the efficiency of chemical etching has been proven in several studies, see e.g. ${ }^{[18]-[20]}$. For instance, using a solution consisting of a mixture of hydrofluoric acid (HF), nitric acid (HNO3) and distilled water reduces the surface arithmetic roughness from $40 \mu \mathrm{m}$ to $11 \mu \mathrm{m}$ after 120 min etching.

This study aims at shedding light on the mechanical properties of corrugated single struts built by powder bed additive manufacturing. It should allow their future use as constitutive elements of complex lattice structures. The impact of surface defects on their mechanical behavior, and in particular, on their ability to unfold is investigated. A dedicated procedure is developed in order to produce corrugated struts with varied surface defects while keeping all other geometrical parameters unchanged. In-situ micro-CT tensile tests are performed to characterize the struts mesostructure during straining and its link with its mechanical behavior.

The effect of macroscopic roughness on the unfolding behavior of corrugated struts is determined. An analysis of the harmfulness of surface defects is then performed. In order to discuss the experimental results, a model describing the probability of failure as a function of the unfolding ratio for several surface aspects is developed. This model is based on the severity of defects and their location along the corrugation. 


\section{Materials and methods}

\subsection{Sample design and manufacturing}

Corrugated single struts generated by a circular cross-section along a sinusoidal shape have been designed as shown in Figure 1. They consist of a gauge length $L 0$ of $18 \mathrm{~mm}$ with a sinusoidal shape of constant maximum amplitude $\mathrm{h}=2 \mathrm{~mm}$ and a period $\mathrm{P}=12 \mathrm{~mm}$. Transition zones of half a period length and an amplitude two times smaller has been added at both ends of the gauge length. Such zones exhibit a reduced curvature to link the gauge length and the grip zone.

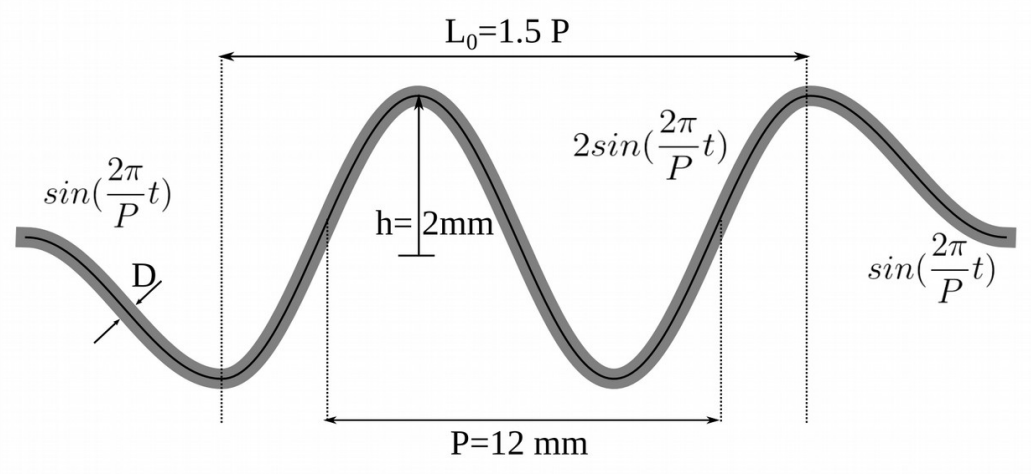

(a)

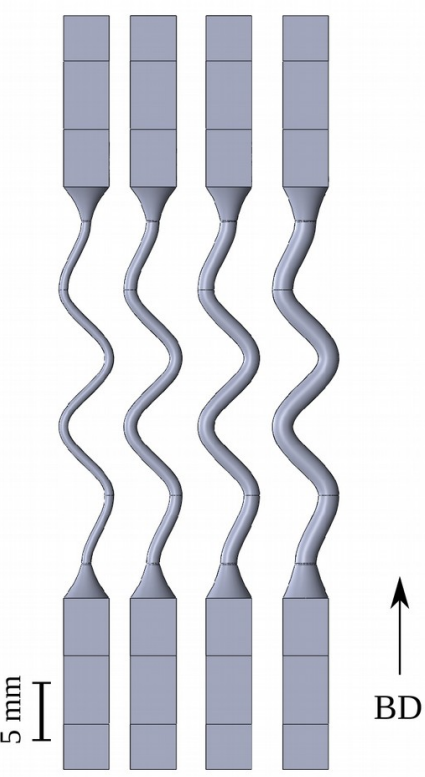

(b)

Figure 1: (a) Longitudinal cross-section showing the parameters of the corrugation used in this study. (b) CAD view of the samples with varying diameters from $0.8 \mathrm{~mm}$ to $1.8 \mathrm{~mm}$.

Samples with nominal diameter D of $0.8 \mathrm{~mm}, 1.1 \mathrm{~mm}, 1.4 \mathrm{~mm}$ and $1.8 \mathrm{~mm}$ have been produced as shown in Figure 1 (b). The corrugated zone is linked to the grips with a fillet. Struts with a diameter of $0.8 \mathrm{~mm}$ will remain in their as-built conditions whereas struts with larger diameters will be chemically etched for different durations to achieve a target diameter of $0.8 \mathrm{~mm}$ with various surface roughnesses. The diameter was chosen so as to cover a wide range of unfolding ratios. On the one hand, Plancher et al. ${ }^{[12]}$ showed that Ti6Al4V corrugated structures with a roughness of about $25 \mu \mathrm{m}$ and with an amplitude of $2 \mathrm{~mm}$ and a period of $12 \mathrm{~mm}$, a local thickness of $1 \mathrm{~mm}$ can be completely unfolded behavior. On the other hand, preliminary tests showed that as-built corrugated struts with a $0.8 \mathrm{~mm}$ diameter fail during early stages of unfolding. A target diameter of $0.8 \mathrm{~mm}$ was thus selected. For each condition, two samples are produced for reproducibility (A and B). 
Parts are manufactured with an E-PBF A1 machine supplied by ARCAM A.B. The raw material used is Ti6Al4V ELI powder produced by gas atomization. It has a spherical shape with a unimodal size distribution between $45 \mu \mathrm{m}$ and $100 \mu \mathrm{m}$. The chemical composition of the powder, as provided by the supplier, is reported in Table 1. The standard beam and scan strategy referenced as "Melt theme" by the machine supplier is chosen for this study. The thermal cycle of the E-PBF process integrates a preheating stage prior to selective melting of every single layer. For the Ti6Al4V alloy, this preheating is carried out at roughly $700^{\circ} \mathrm{C}$. The consequences of this pre-heating are (i) a reduction of the thermal gradients; and (ii) an in-situ heat treatment for the underneath layers inducing a substantial decrease of the residual stresses in the built parts in comparison with parts made by L-PBF ${ }^{[21][22]}$. Figure 2 shows a 3D rendering of an as-built $0.8 \mathrm{~mm}$ diameter corrugated strut (grey) with its internal pores (red). The voxel size is $10 \mu^{3}$. The porosity is lower than $0.1 \%$ and pores are spherical which shows no lack of fusion. Previous studies with a higher resolution (voxel size of $2 \mu \mathrm{m}^{3}$ and 2,5 $\mu \mathrm{m}^{3}$ ) showed that the use of this theme to produce thin parts allows a controlled porosity fraction lower than $0.1 \%{ }^{[16][23]}$. In this

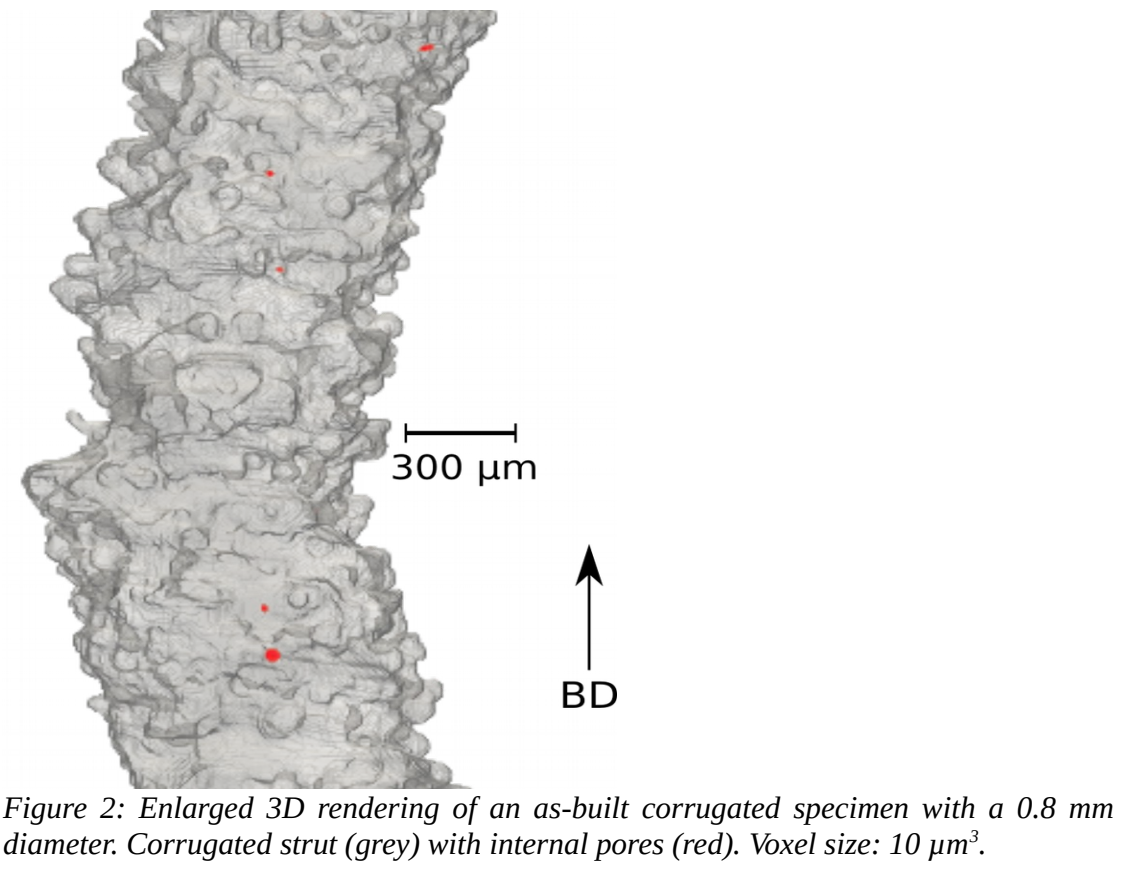

study, the influence of porosity will thus not be taken into account. Only the effect of surface defects is investigated.

Corrugated struts are manufactured vertically (see Building Direction: BD, Figure 1.a). The influence of build orientation on the surface quality of struts manufactured by E-PBF has been already investigated ${ }^{[16][17][24]}$. Compared to L-PBF, E-PBF allows a larger freedom of struts build orientations ${ }^{[16][25]}$. Horizontal struts show poorer surface quality than vertical struts but vertical struts present more detrimental notches than inclined ones. Vertical struts exhibit also better dimensional accuracy than horizontal ones. Indeed, a previous study ${ }^{[16]}$ showed that "over-melting" occurs when manufacturing horizontal struts because of the heat release which can only happen through the powder bed. This tends to over-size the strut along the build direction due to heat accumulation on the down-facing zone. Based on this previous observations, the corrugated struts in this study exhibit surfaces ranging from vertical to $45^{\circ}$ inclined surfaces.
$\mathrm{Al}$ $\mathrm{V}$
$\mathrm{Fe}$
$\mathrm{C}$
$\mathrm{O}$
N
6.47
3.98
0.20
0.02
0.08
0.02
$\mathrm{H}$
Y
$\mathrm{Ti}$

Table 1: Chemical composition of the Ti6Al4V powder. 


\subsection{Chemical etching and micro-CT inspection}

The strategy developed here is to produce corrugated struts with the same diameter $(0.8 \mathrm{~mm})$ but with different surface qualities. To do so, samples produced by E-PBF with different CAD diameters are subsequently etched for different times in an etchant consisted of 5\% HF (48\%), 16\% HNO3 (70\%) and $79 \%$ distilled water. Samples with diameters of 1.1, 1.4 and $1.8 \mathrm{~mm}$ are etched for various durations until reaching the target size: a $0.8 \mathrm{~mm}$ diameter.

The predictive determination of this etching duration is not straightforward. It is indeed not possible to rely on previous studies ${ }^{[18]}$ since the etchant concentration and the geometry of the samples are different. In order to find the suitable etching duration, micro-CT is performed on corrugated samples in their as-built state and after various etching duration. An Easytom XL laboratory tomograph (RX Solutions $\left.{ }^{\circledR}\right)$ with a tungsten source and a flat panel detector is employed. The source tension and intensity are set to $80 \mathrm{kV}$ and $125 \mu \mathrm{A}$. A fast scan is performed with 300 projections at a voxel size of $8.5 \mu^{3}$ in roughly 5 minutes. Volumes are reconstructed using standard filtered back-projection with the software X-act by RX-solution ${ }^{\circledR}$. The reconstructed volumes are then filtered and thresholded. Corrugated struts present less than $0.1 \%$ porosity. For each sample, the variation of cross-sectional surface in the gauge zone is analyzed and compared to the one of the as-built $0.8 \mathrm{~mm}$ sample. For sake of simplicity, the area is calculated on a transverse cross-section normal to the build direction. The surface roughness of the struts does not allow to rely on the target diameter in order to determine the required etching time. An example of the developed method for a sample with an initial diameter of $1.8 \mathrm{~mm}$ is shown in Figure 3(a). The surface variation at each slice of the volume inside the gauge zone is plotted. The theoretical profile obtained on the CAD is presented in black. Because the crosssection is not normal to the neutral fiber of the strut, the surface evolution is not constant. The maximal and minimal cross-section areas their corresponding location within a strut of Figure 3 (b) is also represented. It corresponds to the position of maximal curvature and minimal curvature. The surface is maximal when the neutral fiber of the strut is at $45^{\circ}$ from the cross-section i.e. the crosssection area is the most elliptic. The cross-section area is minimal when the cross-section is the most circular. The red curve represents the targeted reference of the as-built $0.8 \mathrm{~mm}$ diameter strut. The surface variation for the as-built state as well as after $30 \mathrm{~min}, 40 \mathrm{~min}$ and $50 \mathrm{~min}$ of etching are also shown in blue in Figure 2 (a). It can be seen that for 50 min of etching, the cross-section surface variation of the $1.8 \mathrm{~mm}$ sample reaches the one of the $0.8 \mathrm{~mm}$ as-built sample. 


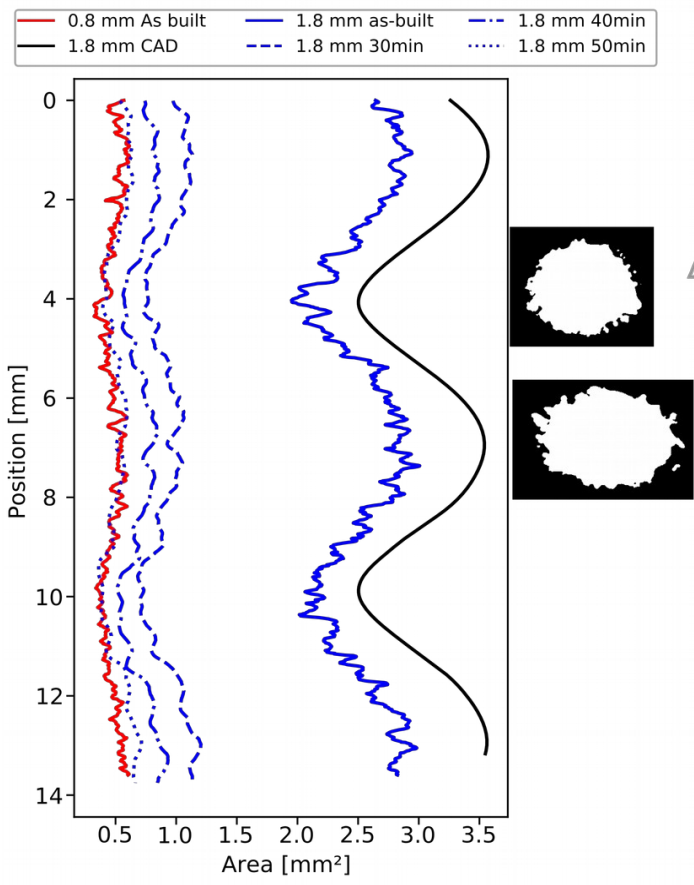

(a)

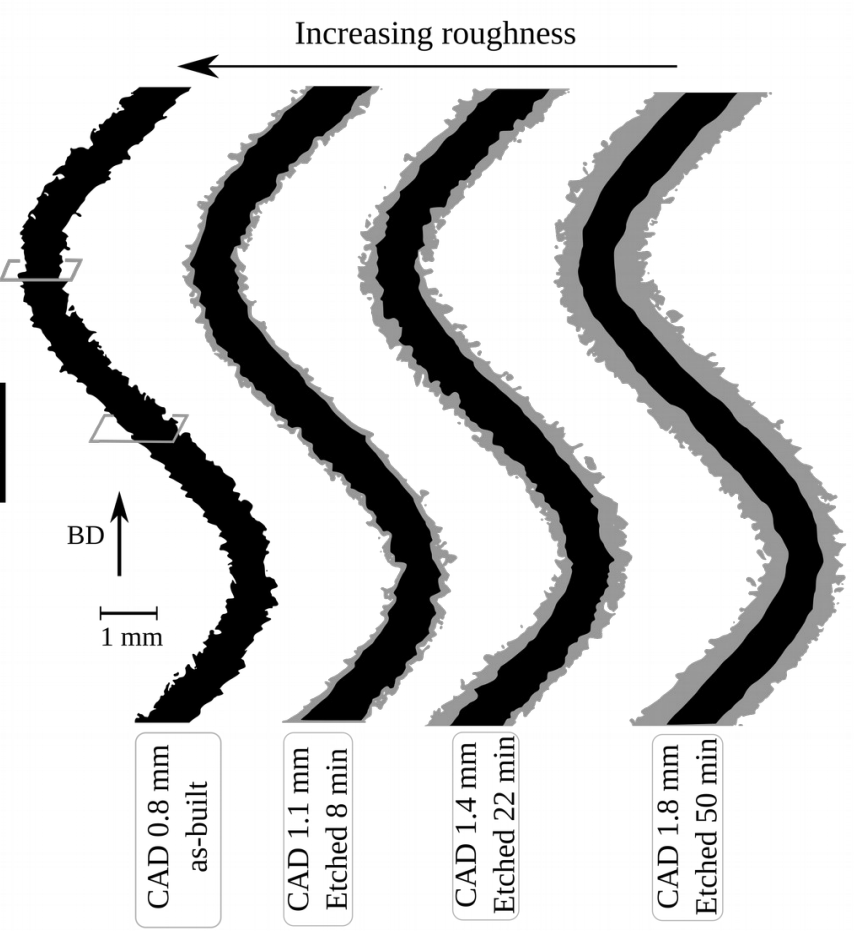

(b)

Figure 3: (a) Variation of strut's transverse cross-section in the gauge zone for an initial diameter of $1.8 \mathrm{~mm}$ in its as-built state and after $30 \mathrm{~min}, 40 \mathrm{~min}$ and $50 \mathrm{~min}$ of etching (blue). Comparison with as-built $0.8 \mathrm{~mm}$ sample (red) and with theoretical profile for $1.8 \mathrm{~mm}$ CAD (black).(b) Mid-plane section of each configuration $(0.8 \mathrm{~mm}, 1.2 \mathrm{~mm}, 1.5 \mathrm{~mm}$ and $1.8 \mathrm{~mm})$ before (grey) after etching (black). Cross-section areas are similar while roughness is varied.

The same procedure has been applied to struts with an initial diameter of $1.1 \mathrm{~mm}$ and $1.4 \mathrm{~mm}$. The etching duration to obtain a similar variation of surface throughout the strut is $8 \mathrm{~min}, 22 \mathrm{~min}$ and 50 min for an initial diameter of respectively $1.1 \mathrm{~mm}, 1.4 \mathrm{~mm}$ and $1.8 \mathrm{~mm}$. Their shape before and after chemical etching is shown in Figure 3 (b). Chemical etching allows the global roughness to be reduced.

In order to quantify roughness, an in-house ImageJ routine has been employed to extract the position of the lower profile throughout the strut (in the $30 \mathrm{~mm}$ corresponding to the gauge length and both transition zones) as shown in Figure 4 (a) in red. The profile is then filtered with a low-pass filter to determine its baseline (green line). The subtraction of the profile with its baseline produces a normalized profile, i.e. the profile of a straight sample having the same roughness (black line in Figure 4 (a)). Arithmetic roughness is calculated from this profile as follows:

$$
R a=\frac{1}{L} \int_{0}^{L}|r(l)| d l \approx \frac{1}{L} \sum_{0}^{L}|r(l)|
$$

The determination of a notch-like defect depth from a 3D image is not straightforward. In this study, it is determined based on the minimum distance from the surface to the neutral fiber. At each slice (cross-section normal to the build direction) the neutral fiber point is determined as the center of mass (red in Figure 4 (b)). In parallel, a 3D Euclidean distance map is also performed on the strut. Each pixel is assigned the value of its minimum distance to the surface. The value corresponding to the distance map at the position of the neutral fiber for each slice is measured and denoted $\delta \mathrm{i}$. It corresponds to the 3D minimal distance from the neutral fiber to the surface (green in Figure 4 (b)). 
The notch depth variation is defined as the difference between the average 3D minimum distance denoted $<\delta \mathrm{i}>\mathrm{i}$ and the 3D minimum distance to the surface throughout the strut, $\delta \mathrm{i}$ :

$$
a_{i}=\left\langle\delta_{i}\right\rangle_{i}-\delta_{i}
$$

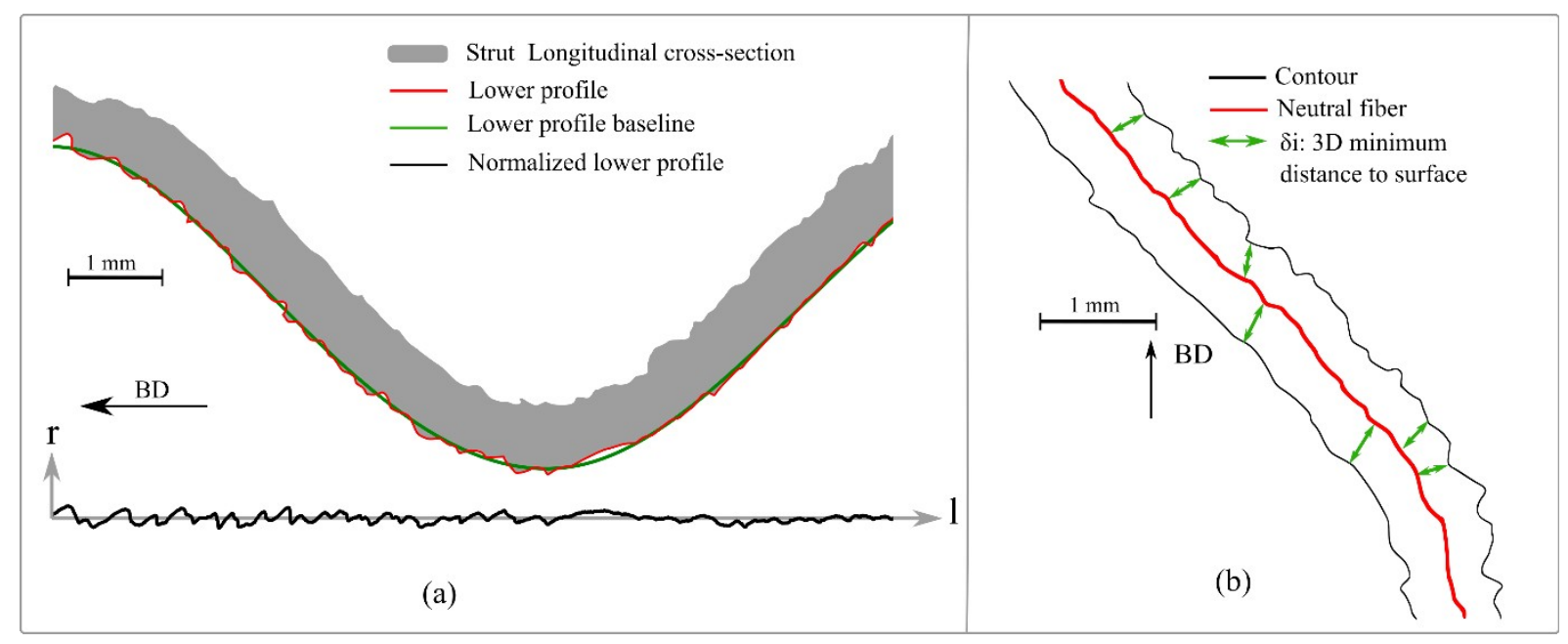

Figure 4: (a): 2D view of the longitudinal cross-section describing the methodology for roughness calculation including the normalization with the baseline. (b): $2 D$ schematic of the 3D minimum distance determination between the neutral fiber and the surface.

\subsection{In-situ tensile testing}

In-situ tensile testing is performed using the same Easytom XL laboratory tomograph (RX Solutions $\left.{ }^{\circledR}\right)$. It is equipped with a micro-focus W source and a flat panel detector. The source tension and intensity is kept constant at $120 \mathrm{kV}$ and $83 \mu \mathrm{A}$. The beam is filtered with a copper plate of $0.5 \mathrm{~mm}$. The voxel size is set to $10 \mu \mathrm{m}^{3}$. The visualization of the entire gauge length thus requires a helical scan with 1200 projections and 4 turns over a translation of $51 \mathrm{~mm}$. The frame rate is set to 6 frames per second and for each frame, the resulting projection is averaged over two images. The resulting scan duration is around 7 minutes. Volumes are reconstructed using standard filtered back-projection with the dedicated software edited by RX-solution ${ }^{\circledR}$. The generated volumes are filtered with a 3D median filter of radius one voxel. They are then binarized using a constant threshold value.

An in-house tensile device has been used for in-situ mechanical tests. An engine controls the motion of the bottom grip while the top grip position remains static. A $500 \mathrm{~N}$ load sensor records force during the experiments. A pre-load of $5 \mathrm{~N}$ is applied and then the displacement is applied at a speed of $10 \mu \mathrm{m} / \mathrm{s}$. Scans are taken at specific bottom grip displacements, also called raw displacements: $0.5 \mathrm{~mm}, 1 \mathrm{~mm}$, $1.5 \mathrm{~mm}, 2.5 \mathrm{~mm}, 3.5 \mathrm{~mm}, 4.5 \mathrm{~mm}, 5.5 \mathrm{~mm}, 6.5 \mathrm{~mm}$. For each scan, the true elongation of the gauge length is also calculated based on the obtained images. It is carried out by following in 3D two surface points located at the extremity of the gauge length. The raw displacement is then corrected to fit with those discrete values of displacement calculated at each scan. This corrected displacement is used to calculate the unfolding ratio. A purely geometric criterion is defined. The initial arc length of the sinusoidal neutral fiber covering the gauge length and both transition zones (explained in 1.1) is calculated. The unfolding ratio is calculated so as it reaches $100 \%$ when the gauge length attains the initial arc length. 


\section{Results}

\subsection{Cross-section monitoring for reducing surface defects}

The mean area of the struts in the gauge length is shown in Figure 5 (a) with unicolor faces as compared to the initial mean area plot with dotted faces. Chemical etching allowed a global reduction of the cross-section area to reach a comparable cross-section as in the as-built samples with a $0.8 \mathrm{~mm}$ diameter.

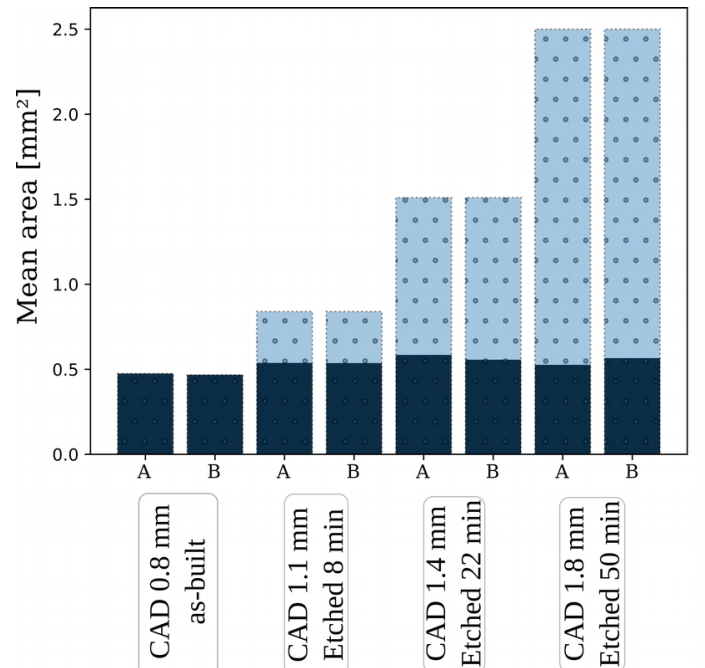

(a)

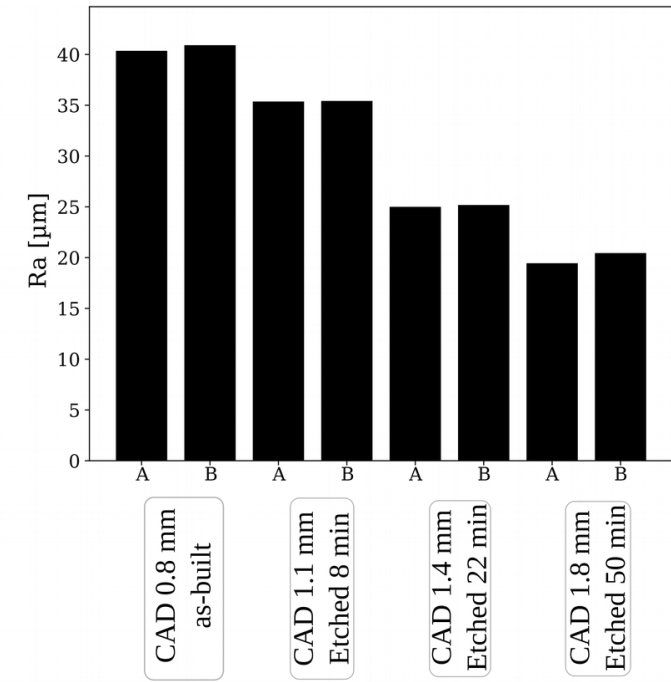

(b)

Figure 5: (a): Average cross-section of the samples before (dotted face) and after chemical etching (unicolor face). (b): longitudinal arithmetic roughness of the samples after etching.

Figure 5 (b) shows the longitudinal arithmetic roughness calculated for each sample. The effect of chemical etching on the initial roughness is clearly visible. The raw arithmetic roughness is in the range of $40 \mu \mathrm{m}$ whereas after $50 \mathrm{~min}$ of chemical etching it decreases down to roughly $20 \mu \mathrm{m}$. Several studies reported similar values of raw arithmetic roughness for E-PBF parts, see ${ }^{[16][20][26]}$. Although roughness is reduced when increasing chemical etching, residual notch-like defects remain as can be seen in Figure 3 (b). This has been already shown in the literature, see e.g. ${ }^{[18][27]}$.

Samples will now be denoted from their initial average arithmetic roughness Ra as shown in Figure 5 (b). For instance, the as-built $0.8 \mathrm{~mm}$ struts are then called "Ra $50 \mu \mathrm{m}$ ", whereas the struts with an initial diameter of $1.4 \mathrm{~mm}$ and a 22 min etching are called "Ra $25 \mu \mathrm{m}$ ".

\subsection{Mechanical behavior}

For each sample, uniaxial in-situ tensile tests have been carried out until failure. Figure 6 shows the tensile curve for a corrugated strut with $\mathrm{Ra}=20 \mu \mathrm{m}$. The force versus unfolding ratio curve exhibits unusual behavior. At around $20 \%$ of unfolding, struts start to locally yield in the zones of large curvature. It produces a change of the curve slope. At around $95 \%$ of unloading, the slope rises due to a complete loading of the unfolded strut. Similar behavior has been observed in a numerical study of such corrugated struts [12]. 

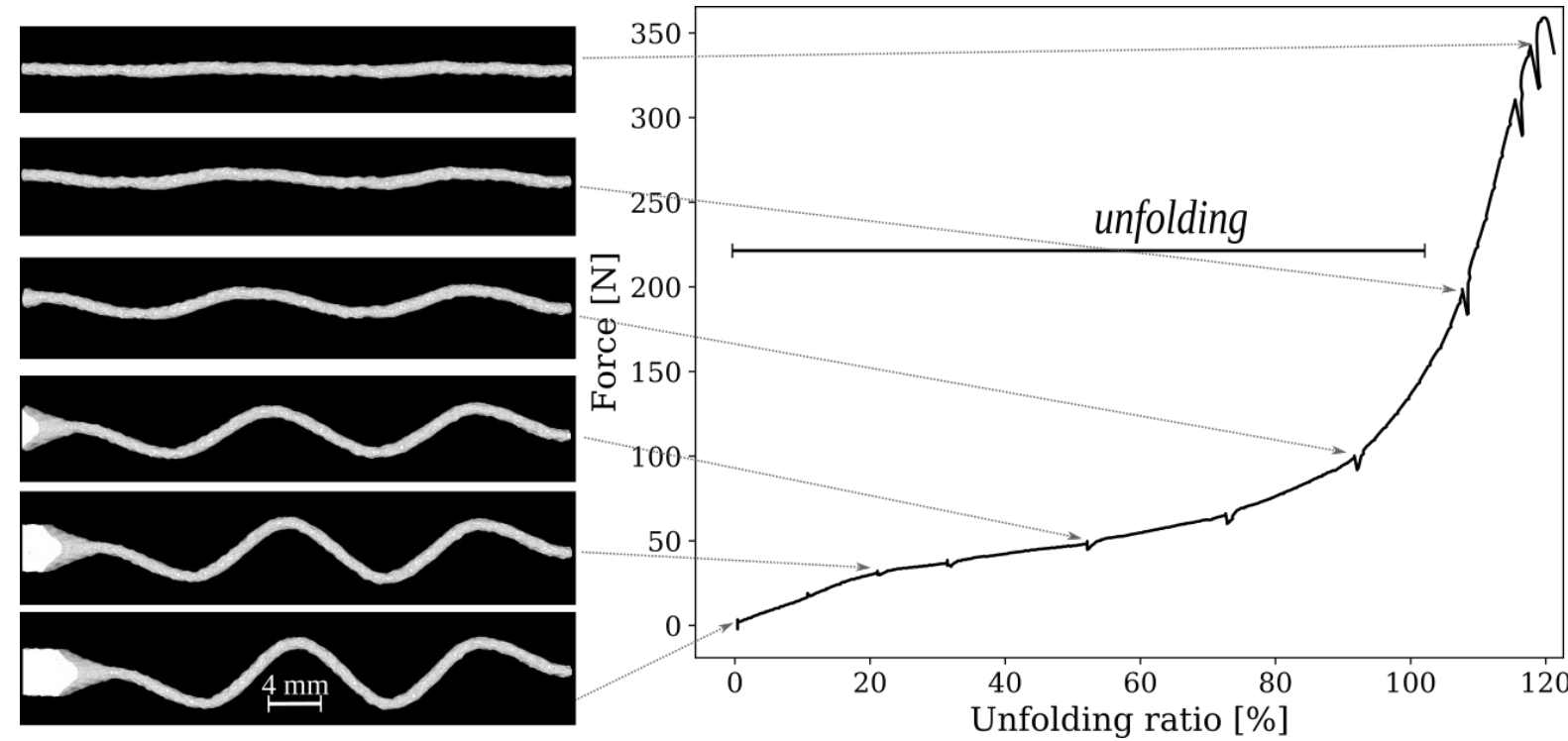

Figure 6: Example of tensile test results on sample B with $\mathrm{Ra}=20 \mu \mathrm{m}$. Tensile curve and corresponding $3 D$ views of the strut during unfolding.

As explained earlier, scans have been obtained at given displacements. During the scans, relaxation is observed, see Figure 6. The corresponding 3D view during unfolding is shown on the left side. It shows a complete unfolding for this strut slightly after an unfolding ratio of $100 \%$. As explained in 1.3, the calculation of the unfolding ratio is purely geometric. However, elastic and plastic deformations occur during loading. It explains why the strut is not completely unfolded at an unfolding ratio of $100 \%$.

Figure 7 exhibits the resulting force as a function of strut's unfolding for the eight samples (four different roughnesses and two samples by conditions). It shows very different unfolding behaviors between as-built samples and etched ones. As-built samples unfold barely whereas samples that undergo long etching steps unfold completely. All the etched samples display the same level of force whatever the etching time. The only difference is the maximum unfolding that is reached. As-built samples exhibit slightly lower levels of force. This can be attributed to the difference in initial mean area as shown in Figure 5 (a). Indeed, they exhibit slightly lower mean surface area resulting in lower levels of force. 


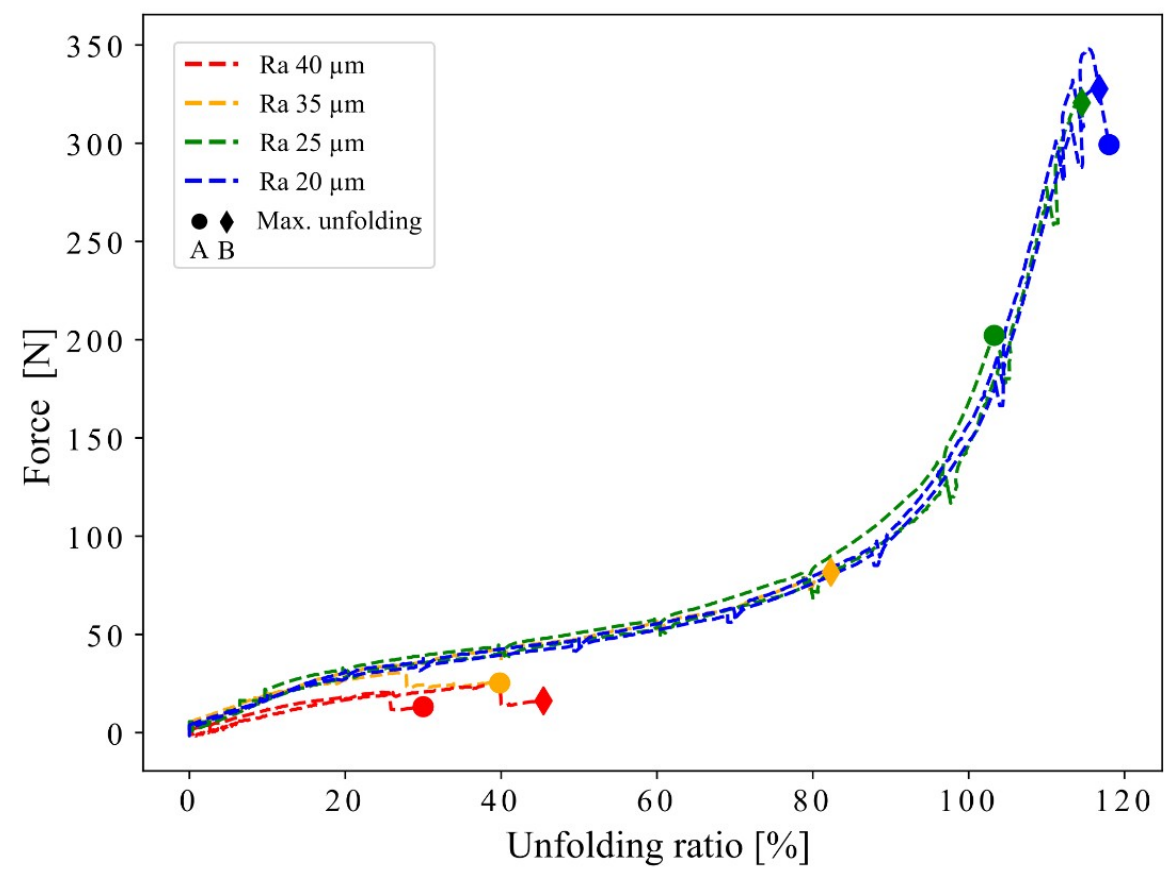

Figure 7: Tensile curves of samples showing different unfolding behaviors

The influence of surface roughness reduction on the unfolding of corrugated struts is significant. However, for a given strut roughness, strong variability remains between both samples.

\subsection{Effect of initial roughness on unfolding}

Figure 8 shows the unfolding ratio at failure of the samples as a function of their arithmetic roughness. The arithmetic roughness affects largely the maximal unfolding capability under tensile loading. A smoother surface allows larger unfolding since there are less crack initiation sites. However, Figure 7 shows also a large difference of unfolding ratio for samples having the same roughness, especially for intermediate roughness values. The global arithmetic roughness of the sample is thus not a criteria allowing a fully predictive behavior of corrugated struts. The location of the most detrimental surface defects as a function of the local curvature and stress state needs to be deeply investigated. 


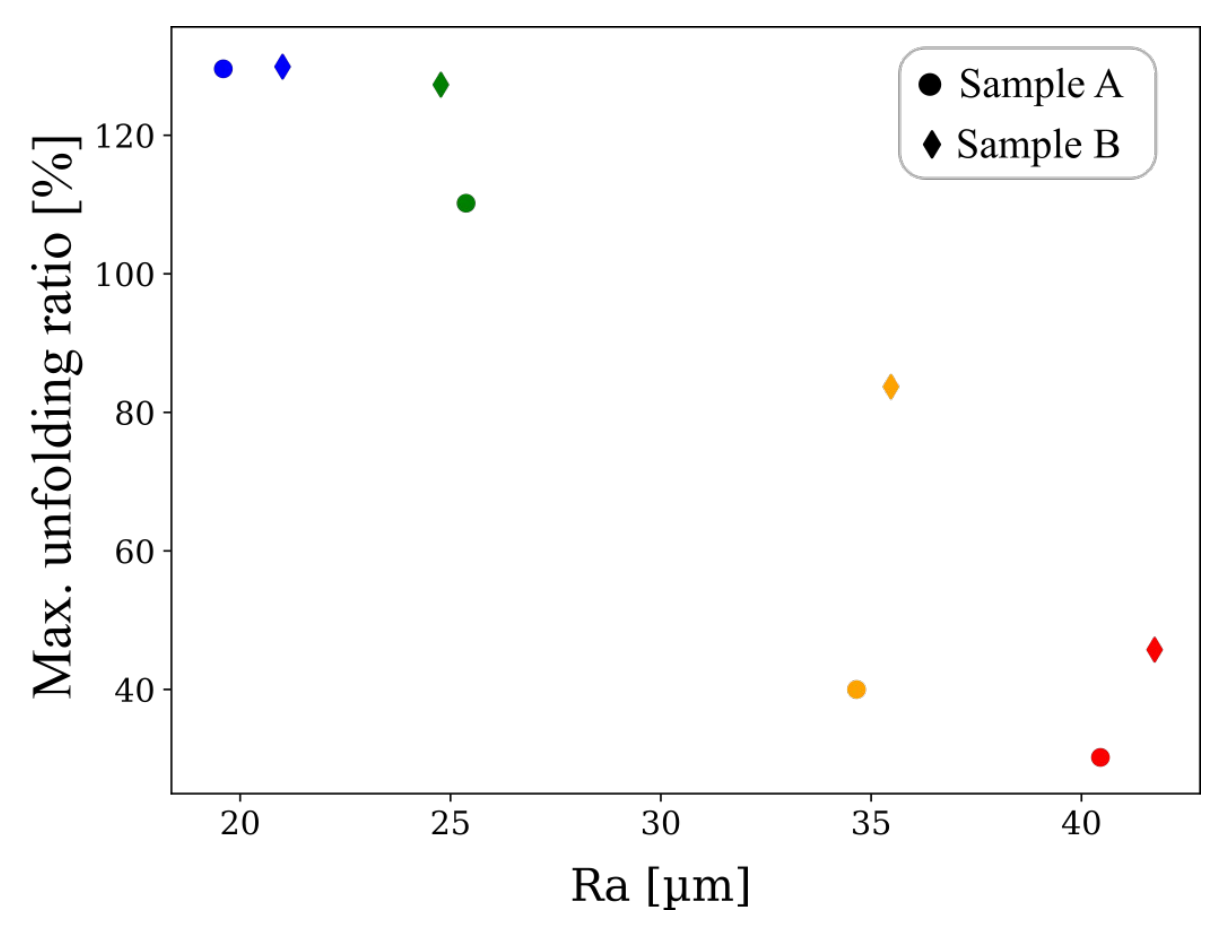

Figure 8: Variation of maximal unfolding ratio as a function of the arithmetic roughness of the samples.

\subsection{Local surface defects detection}

The ability for a corrugated strut to unfold cannot be fully predicted based on its roughness. Due to the corrugated geometry, zones with a larger curvature are the most loaded. The presence of a surface defect in this zone is, therefore, more detrimental than anywhere else. In this study, notches are the only surface defects considered. The stress concentration factor of a notch-like defect depends on its depth and its tip radius. However, due to the resolution employed in this work, the radius of curvature at the tip of the notches cannot be properly assessed. The notch depth is taken here as the only criterion to categorize the criticality of a notch.

Figure 9 represents the variation of the notch depth throughout the struts for all the tested conditions in this work. It is rather clear that struts with lower arithmetic roughness exhibit less notch-like surface defects. However, even for a low arithmetic roughness, notches as deep as $60 \mu \mathrm{m}$ can remain. Their number is reduced and thus their probability to be located in a critical zone (i.e. with a large curvature) is limited.

For an arithmetic roughness of about $35 \mu \mathrm{m}$, Figure 8 shows a large difference of unfolding capabilities between samples A and B. Figure 9 helps to rationalize this difference. For sample A, a deep notch (depth larger than $60 \mu \mathrm{m}$ ) is located in a zone of largest curvature. At this location, large local stress values will occur during unfolding. This notch will, therefore, induce premature failure as compared to sample B for which deepest notches are not located in the zones of maximum stress. 


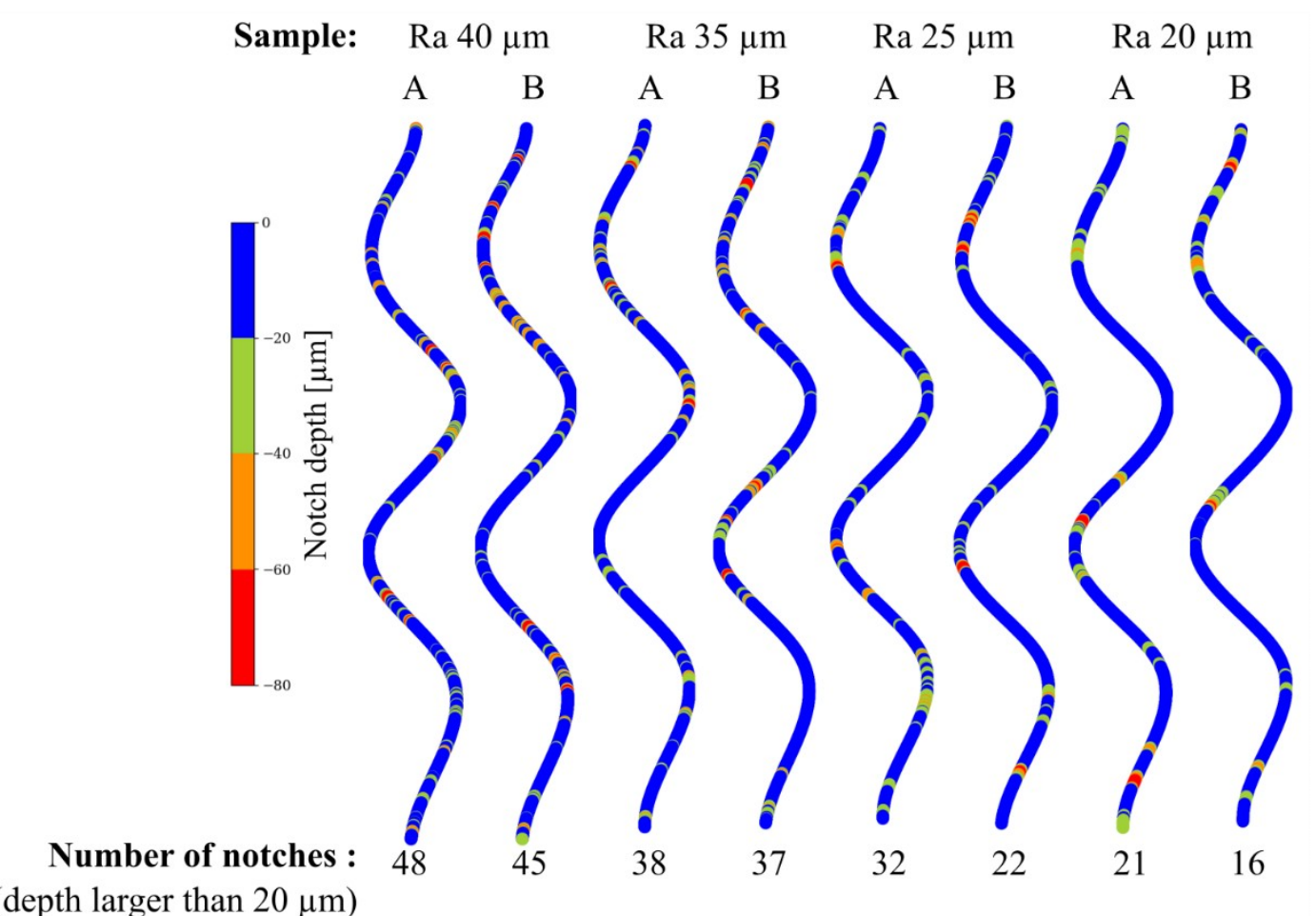

Figure 9: Distribution of surface defects within the corrugated struts. Colors represent the depth for each notch. The number of notches for each sample is placed underneath.

\section{Discussion}

\subsection{Critical notch determination}

A critical notch classification has been proposed by Tammas-Williams et al. ${ }^{[28]}$ using several criteria. They suggested ranking the defects (bulk or surface) based on their size, maximum stress intensity factor, or their morphology and proximity to surface. In the present study, we account for surface defects only. Considering such surface defects, Tammas-Williams et al. ${ }^{[28]}$ evaluated their harmfulness by measuring their stress intensity factor, size (square root of maximum area normal to the stress), proximity to other defects... For sure, the relevant criteria to determine the harmfulness of notches is not only their size but also their shape. However, surface defects that initiate crack have a depth of 50$60 \mu \mathrm{m}$. The depth of a harmful surface defect has been assessed in several studies on Ti64 samples produced by E-PBF. It was shown that the crack responsible for failure has a minimum intrinsic size of around $60 \mu \mathrm{m}{ }^{[17][28]}$. The resolution of the micro-CT in the present study does not allow a correct determination of notch shape. That's why, in the present study, a notch depth of $60 \mu \mathrm{m}$ is taken as the critical value to evaluate the harmfulness of surface defects. For each sample, notches with a depth larger than this value (red in Figure 9) are counted. Table 2 shows their frequency depending on the sample roughness. The presented value is a mean value of both samples (A and B) for each configuration.

\begin{tabular}{lcccc} 
Arithmetic roughness $[\mu \mathrm{m}]$ & 40 & 35 & 25 & 20 \\
\hline Critical notches frequency $\left[\mathrm{n}^{\circ} / \mathrm{mm}\right]$ & 0.23 & 0.16 & 0.10 & 0.07
\end{tabular}

Table 2: Critical notches frequency calculated as the mean value of both samples in each configuration.

The frequency of harmful notches decreases when decreasing the strut arithmetic roughness. 


\subsection{Position of notch-like defects along the corrugations}

During unfolding, the stress state is heterogeneous in the corrugated strut as explained in detail in a previous study by Plancher et al. ${ }^{[12]}$. They developed a 2D FEM model on a single period integrating a large-displacement and large-rotation framework with plane stress boundary conditions. Plane stress assumption allowed the use of a 2D model after comparison with a full 3D calculation. They focused on the stress state in the maximum strain zone, i.e. the one with large curvature to understand the unfolding behavior of such corrugated struts.

A new simulation adapted to the corrugation parameters of the current study and based on the framework proposed by Plancher et al. ${ }^{[12]}$ is developed. In order to determine the most harmful notch positions during unfolding, zones with large tensile stress are extracted. Note that the model is 2D while current struts present a circular cross-section. Such approximation is crude but will be fine enough to capture the order of magnitude. A threshold value of the tensile stress inducing crack propagation is chosen as $950 \mathrm{MPa}$. It is the yield point of the constitutive material of the strut (from the powder supplier ${ }^{[29]}$ ). For sake of simplicity, it is assumed that zones where the strut is already yielding are critical zones where the presence of a large notch can induce failure. Figure 10 shows the critical fraction of the strut exhibiting tensile stress values larger than $950 \mathrm{MPa}$ during unfolding. At several unfolding ratios, an image of the stress field higher than this value resulting from the finite element simulation is shown. As explained in 1.3, the determination of the unfolding ratio as a function of the displacement is carried out assuming a $100 \%$ unfolding when the total length corresponds to the initial arc length of the strut. This criteria is purely geometric and does not take into account local plastic strain and elastic strain. It explains why the strut is not completely unfolded for an unfolding ratio of $100 \%$.

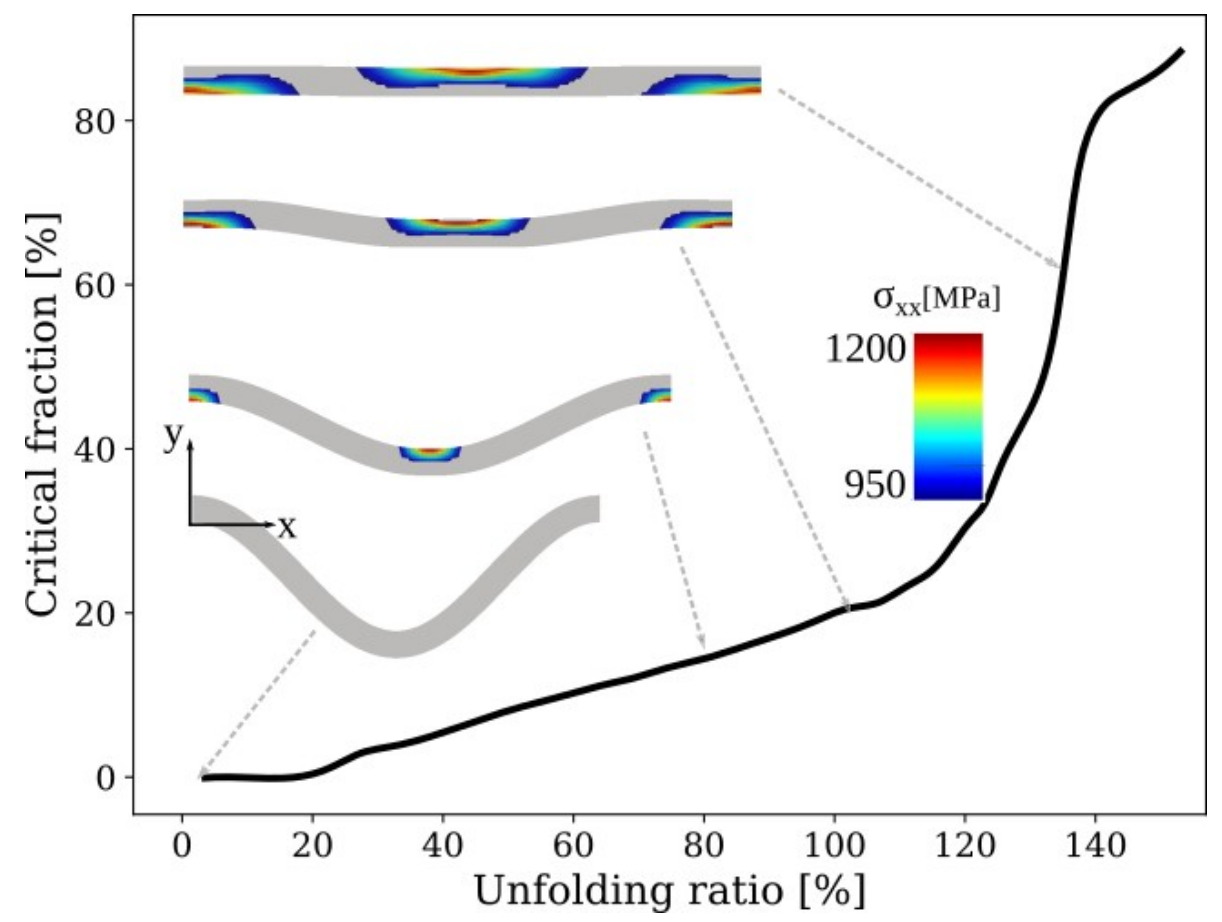

Figure 10: Variation of critical surface fraction of a single period of a corrugated strut corresponding to zones with a tensile stress larger than $950 \mathrm{MPa}$. Corresponding views of such zones at different unfolding stages have been superimposed using FEM results.

The critical fraction is kept to 0 until an unfolding of around $20 \%$ when the strut is elastically loaded. This critical fraction increases linearly during unfolding until unfolding reaches $100 \%$. The zones sustaining high tensile stress are in the internal part of the curvatures. The critical fraction then 
increases rapidly corresponding to the increase of the tensile stress all along the strut. The knowledge of the critical fraction of the struts during unfolding and the harmful notch frequency allows the prediction of failure for each configuration during unfolding.

\subsection{A probabilistic model to predict strut failure during unfolding}

A simple model for predicting the probability of failure during the unfolding of corrugated struts as a function of their initial surface quality is proposed. It is based on the assumption that failure occurs if a harmful notch (i.e. with a depth larger than $60 \mu \mathrm{m}$ ) is located within a region sustaining large tensile stresses (typically where $\sigma_{X X}$ is larger than $950 \mathrm{MPa}$ ). Since this model is based on a critical notch density (section 3.1), the notches are supposed to be regularly distributed along the corrugated strut.

The probability of failure at a given unfolding ratio is calculated as follows:

$$
P(i)=C_{f} \cdot L \cdot N_{f}
$$

where $C_{f}$ is the critical surface fraction (as presented in Figure 10), $L$ is the gauge length and $N_{f}$ is the notch frequency as defined in Table 2 . $\mathrm{C}_{\mathrm{f}}$ varies with the unfolding ratio whereas $\mathrm{N}_{\mathrm{f}}$ depends on the arithmetic roughness.

Figure 11 presents the probability of failure as a function of the unfolding ratio for corrugated struts with different surface roughness. It shows that for struts with arithmetic roughness larger than $35 \mu \mathrm{m}$, struts will fail before complete unfolding whereas for struts with an arithmetic roughness of $20 \mu \mathrm{m}$, the probability of failure before complete unfolding is less than $40 \%$. Dots and diamonds correspond to the experimental values of maximal unfolding for each sample. The developed model captures rather well the dispersion of maximal unfolding for each configuration.

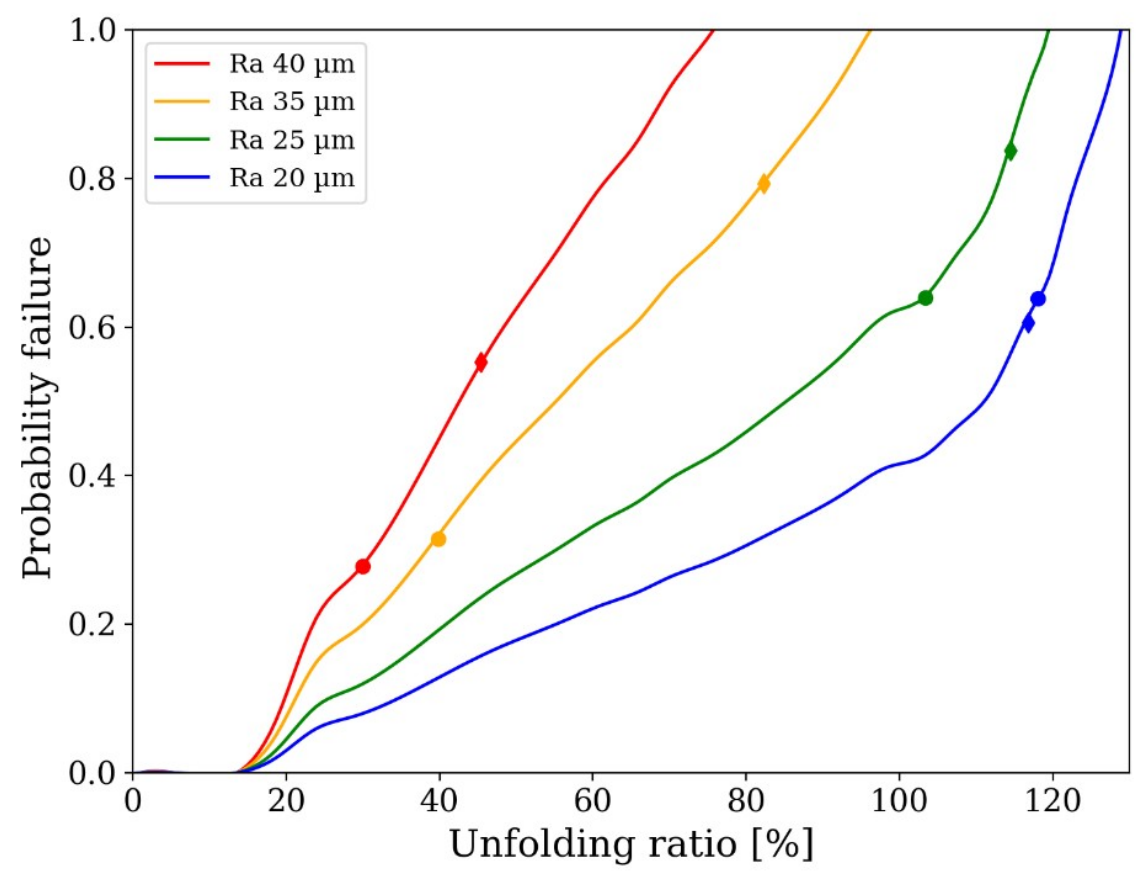

Figure 11: Model for the prediction of the probability of failure as a function of unfolding for struts with different surface roughness. Dots and diamonds represent experimental values of unfolding for A and B samples respectively for each initial roughness. 
Although being based on a 2D simulation and using questionable values of critical notch depth and tensile stress, this simple model gives promising trends for understanding the unfolding variability of struts produced by powder bed additive manufacturing. It brings valuable knowledge for a future use of such corrugated struts inside a global lattice structure. The upgrading of this model is under development to validate its generalization to other geometry and size of corrugated struts.

\section{Conclusion}

This study analyzes the influence of surface defects on the unfolding ability of corrugated struts. Such struts can exhibit promising mechanical behavior when integrated within lattice structures. Their shape induces a macroscopic stress-concentration at location of maximal curvature. It is kept constant in this study by holding the corrugation parameters unchanged. The surface roughness produces a mesoscopic stress-concentration that is evaluated here.

- A dedicated experimental procedure has been developed in order to produce corrugated struts with varied roughnesses while keeping all other geometrical parameters unchanged. It is based on a combination of Electron Beam Powder-Bed Fusion additive manufacturing and postprocessing using chemical etching assisted by micro-CT. Struts are etched for 8, 22 and 50 min for initial diameters of 1.1, 1.4 and $1.8 \mathrm{~mm}$ in order to achieve the same final crosssection but with different surface roughness (from $\mathrm{Ra}=40 \mu \mathrm{m}$ to $\mathrm{Ra}=20 \mu \mathrm{m}$ ).

- In-situ micro-CT tensile tests are performed in order to characterize the struts morphology evolution during straining. The maximal unfolding ability of each sample has been measured.

- Samples with large surface roughness fail before complete unfolding whereas samples with a lower surface roughness lead to larger values of unfolding ratio (until complete unfolding). Large differences of maximal unfolding could remain between samples having the same surface roughness.

- The surface roughness is not the appropriate criterion to predict the maximal unfolding of a corrugated strut. A more detailed analysis based on the residual notch-like defects characterization is performed. The maximal unfolding of a corrugated strut is thus related to the harmfulness of the surface notch-like defects (based on their depth), their frequency and position within the sinusoidal shape. A harmful notch located in a zone of large curvature (submitted to large local tensile stress during unfolding) leads to premature failure. On the contrary, a critical notch located in a zone of small curvature (submitted to low tensile stress) is much less detrimental.

- A model based on the defect density and a ratio of "significantly loaded" volume is proposed. It allows the prediction of the probability of failure during unfolding for corrugated struts with different surface roughness. It is validated by experimental results and captures well the dispersion of maximal unfolding for each configuration.

\section{Aknowledgments}

This work was performed within the framework of the Center of Excellence of Multifunctional Architectured Materials "CEMAM" nAN-10-LABX-44-01 funded by the "Investments for the Future Program”.

\section{Conflict of interest}

The Authors declare that there is no conflict of interest. 


\section{References}

[1] Y. Brechet, J.D. Embury, Scr. Mater. 2013, 68, 1.

[2] A.G. Evans, J.W. Hutchinson, M.F. Ashby, Prog. Mater. Sci. 1999, 43, 171.

[3] A.G. Evans, J.W. Hutchinson, N. a. Fleck, M.F. Ashby, H.N.G. Wadley, Prog. Mater. Sci. 2001, 46, 309.

[4] M. Ashby, Scr. Mater. 2013, 68, 4.

[5] T.A. Schaedler, A.J. Jacobsen, A. Torrents, A.E. Sorensen, J. Lian, J.R. Greer, L. Valdevit, W.B. Carter, Science (80-. ). 2011, 334, 962.

[6] D.B. Miracle, Compos. Sci. Technol. 2005, 65, 2526.

[7] W. Xu, D.P. Edwards, X. Wu, M. Stoica, M. Calin, U. Kühn, J. Eckert, K. Xia, Scr. Mater. 2013, 68, 67.

[8] D.J. Lloyd, Scr. Mater. 2013, 68, 13.

[9] M.S. Pham, C. Liu, I. Todd, J. Lertthanasarn, Nature 2019, 565, 305.

[10] M. Fraser, H. Zurob, P. Wu, O. Bouaziz, Adv. Eng. Mater. 2014, 16, 872.

[11] O. Bouaziz, Scr. Mater. 2013, 68, 28.

[12] E. Plancher, L. Héraud, P. Lhuissier, R. Dendievel, D. Fabrègue, J.J. Blandin, G. Martin, Mater. Des. 2019, 166, 107604.

[13] A.D. Dressler, E.W. Jost, J.C. Miers, D.G. Moore, C.C. Seepersad, B.L. Boyce, Addit. Manuf. 2019, 28, 692.

[14] B. Lozanovski, M. Leary, P. Tran, D. Shidid, M. Qian, P. Choong, M. Brandt, Mater. Des. 2019, $171,107671$.

[15] M. Dallago, B. Winiarski, F. Zanini, S. Carmignato, M. Benedetti, Int. J. Fatigue 2019, 124, 348.

[16] M. Suard, G. Martin, P. Lhuissier, R. Dendievel, F. Vignat, J.-J. Blandin, F. Villeneuve, Addit. Manuf. 2015, 8, 124.

[17] T. Persenot, A. Burr, G. Martin, J.-Y. Buffiére, R. Dendievel, E. Maire, Int. J. Fatigue J. 2019, 118, 65.

[18] P. Lhuissier, C. de Formanoir, G. Martin, R. Dendievel, S. Godet, Mater. Des. 2016, 110, 485.

[19] C. de Formanoir, M. Suard, R. Dendievel, G. Martin, S. Godet, Addit. Manuf. 2016, 11, 71.

[20] Y.Y. Sun, S. Gulizia, C.H. Oh, D. Fraser, M. Leary, Y.F. Yang, M. Qian, Jom 2016, 68, 791. 
[21] L.M. Sochalski-Kolbus, E.A. Payzant, P.A. Cornwell, T.R. Watkins, S.S. Babu, R.R. Dehoff, M. Lorenz, O. Ovchinnikova, C. Duty, Metall. Mater. Trans. A Phys. Metall. Mater. Sci. 2015, 46, 1419.

[22] A. Salmi, E. Atzeni, Virtual Phys. Prototyp. 2020, 15, 49.

[23] T. Persenot, A. Burr, R. Dendievel, J.Y. Buffière, E. Maire, J. Lachambre, G. Martin, Materialia 2020, 9, 100589.

[24] X.Z. Zhang, H.P. Tang, M. Leary, T. Song, L. Jia, M. Qian, Jom 2018, 70, 1870.

[25] S.L. Sing, F.E. Wiria, W.Y. Yeong, Robot. Comput. Integr. Manuf. 2018, 49, 170.

[26] A. Pérez-Sánchez, A. Yánez, A. Cuadrado, O. Martel, N. Nuño, Mater. Des. 2018, 155, 106.

[27] T. Persenot, G. Martin, R. Dendievel, J.-Y. Buffiére, E. Maire, Mater. Charact. 2018, 1.

[28] S. Tammas-Williams, P.J. Withers, I. Todd, P.B. Prangnell, Sci. Rep. 2017, 7, 1.

[29] Arcam, Arcam Website 2014, 3. 